\title{
Enhanced genome editing in human iPSCs with CRISPR-CAS9 by co-targeting ATP1a1
}

\author{
Jui-Tung Liu ${ }^{1}$, James L Corbett ${ }^{1}$, James A Heslop ${ }^{1}$, Stephen A Duncan ${ }^{\text {Corresp. } 1}$ \\ ${ }^{1}$ Department of Regenerative Medicine and Cell Biology, Medical University of South Carolina, Charleston, South Carolina, United States \\ Corresponding Author: Stephen A Duncan \\ Email address: duncanst@musc.edu
}

Genome editing in human induced pluripotent stem cells (iPSCs) provides the potential for disease modeling and cell therapy. By generating iPSCs with specific mutations, researchers can differentiate the modified cells to their lineage of interest for further investigation. However, the low efficiency of targeting in iPSCs has hampered the application of genome editing. In this study we used a CRISPR-Cas9 system that introduces

a specific point substitution into the sequence of the $\mathrm{Na}^{+} / \mathrm{K}^{+}$-ATPase subunit ATP1A1. The introduced mutation confers resistance to cardiac glycosides, which can then be used to select successfully targeted cells. Using this system, we introduced different formats of donor DNA for homology-directed repair (HDR), including single-strand DNAs, doublestrand DNAs, and plasmid donors. We achieved a 35-fold increase in HDR when using plasmid donor with a 400 bp repair template. We further co-targeted ATP1A1 and a second locus of interest to determine the enrichment of mutagenesis after cardiac glycoside selection. Through this approach, INDEL rate was increased after cardiac glycoside treatment, while HDR enrichment was only observed at certain loci. Collectively, these results suggest that a plasmid donor with a $400 \mathrm{bp}$ repair template is an optimal donor DNA for targeted substitution and co-targeting ATP1A1 with the second locus enriches for mutagenesis events through cardiac glycoside selection in human iPSCs. 


\section{Enhanced genome editing in human iPSCs with} 3 CRISPR-CAS9 by co-targeting ATP1a1

4

5

6

7

8

9

10

Jui-Tung Liu, James L. Corbett, James A. Heslop, Stephen A. Duncan

Department Regenerative Medicine and Cell Biology, Medical University of South Carolina, Charleston, South Carolina, United States

Corresponding Author:

Stephen A. Duncan

173 Ashley Ave, BSB-657A, MSC 508,

Charleston, South Carolina, 29425, USA

Email address: duncanst@musc.edu

\section{Abstract}

Genome editing in human induced pluripotent stem cells (iPSCs) provides the potential for disease modeling and cell therapy. By generating iPSCs with specific mutations, researchers can differentiate the modified cells to their lineage of interest for further investigation. However, the low efficiency of targeting in iPSCs has hampered the application of genome editing. In this study we used a CRISPR-Cas9 system that introduces a specific point substitution into the sequence of the $\mathrm{Na}^{+} / \mathrm{K}^{+}$-ATPase subunit ATP1A1. The introduced mutation confers resistance to cardiac glycosides, which can then be used to select successfully targeted cells. Using this system, we introduced different formats of donor DNA for homology-directed repair (HDR), including singlestrand DNAs, double-strand DNAs, and plasmid donors. We achieved a 35-fold increase in HDR when using plasmid donor with a $400 \mathrm{bp}$ repair template. We further co-targeted ATP1A1 and a second locus of interest to determine the enrichment of mutagenesis after cardiac glycoside selection. Through this approach, INDEL rate was increased after cardiac glycoside treatment, while HDR enrichment was only observed at certain loci. Collectively, these results suggest that a plasmid donor with a 400 bp repair template is an optimal donor DNA for targeted substitution and co-targeting ATP1A1 with the second locus enriches for mutagenesis events through cardiac glycoside selection in human iPSCs.

\section{Introduction}

Precise genome editing technology provides researchers with a powerful tool to investigate the function of genes in nearly any species. By using programmable nucleases to cut the genome, 
researchers can mutate or correct the genes of interest for further investigation. Although there are still concerns about safety issues, such technologies may pave the way to develop new therapies for cancer treatment or genetic diseases (Cornu et al. 2017; Fu et al. 2013; Hsu et al. 2013; Ihry et al. 2018; Pattanayak et al. 2013).

The most widely used technologies to introduce double-strand breaks (DSBs) are zinc-finger nucleases (ZFNs), transcription activator-like effector nucleases (TALENs), and the RNA-guided cluster regularly interspaced short palindromic repeats-Cas9 (CRISPR-Cas9) system (Joung \& Sander 2013; Sander \& Joung 2014; Urnov et al. 2010). ZFNs and TALENs link endonuclease catalytic domains to DNA-binding proteins to generate DSBs. Alternatively, the CRISPR-Cas9 system uses small guide RNAs that are paired with target DNA to induce DSBs by Cas9. The CRISPR-Cas9 appears to be more flexible, efficient, and easier to design, when compared to the other two systems (Gaj et al. 2013).

Generating DSBs at the target site activates two primary endogenous DNA repair mechanisms in mammalian cells (Valerie \& Povirk 2003). The most frequent repair pathway is non-homologous end joining (NHEJ), which is based on the direct ligation of two DNA ends without the need for a homologous template (Kanaar et al. 1998). This error-prone mechanism can create an insertion or deletion of bases in the genome (INDEL), which may disrupt gene expression by introducing frameshift mutations. This methodology has been an important tool for deleting genes of interest across many species (Bapteste \& Philippe 2002; Belinky et al. 2010; Luan et al. 2013). Homologydirected repair (HDR) is a more precise mechanism that is based on DNA recombination between genomic DNA and the homologous template (Johnson \& Jasin 2001). HDR allows the genomic sequences to be modified based on the introduced repair template. Utilizing HDR machinery for genome editing provides advantages for genome engineering due to the accuracy and predictable nature of the mutation. Numerous studies have applied this genome editing strategy to therapeutic approaches (Deverman et al. 2018; Shim et al. 2017; WareJoncas et al. 2018).

Applying genome-editing tools to human induced-pluripotent stem cells (iPSCs) has provided new directions for regenerative medicine and in vitro disease modeling (Robinton \& Daley 2012). Several studies have successfully generated cell lines to recapitulate genetic diseases using the CRISPR/Cas9 system (Ben Jehuda et al. 2018). Despite the advantages, the efficiency of creating mutations via NHEJ or HDR remains relatively low in human iPSCs (Mali et al. 2013; Wang et al. 2013). Single-stranded DNA oligonucleotides (ssODNs) have been used as repair templates to efficiently introduce single-nucleotide mutations, and double strand DNA donor plasmids are used for fragment insertion via HDR (Chen et al. 2011). However, HDR rates vary depending on cell type and status (Saleh-Gohari \& Helleday 2004). Researchers have proposed strategies to enhance the success rate of genome editing, including cell cycle synchronization, introduction of selection markers, and pre-treatment with small molecule NHEJ inhibitors (Chu et al. 2015; Guo et al. 2018; Yu et al. 2015). Recently, researchers applied Cas9 ribonucleoproteins in combination with AAV- 
79 mediated repair template delivery to increase integration frequency (Martin et al. 2019). Although

80 the efficiency is improved from the original technique, the timing of drug treatment and 81 administration of CRISPR-Cas9 are difficult to control due to the cell cycle variation between cell 82 lines.

84 Cardiac glycosides have been used to treat heart failure by targeting ATP1A1, a subunit of $\mathrm{Na}^{+} / \mathrm{K}^{+}$85 ATPase (McDonough et al. 2002; Smith 1984). With exposure to relatively high concentrations of 86 such drugs, cell viability is reduced via the accumulation of intracellular $\mathrm{Ca}^{2+}$ levels (Belusa et al. 87 2002; Lin et al. 2017). The binding site of cardiac glycosides on ATP1A1 has been identified, and 88 N-terminal amino-acid substitution of ATP1A1 encoded by exon4 (Q118R and N129D) is 89 sufficient to confer drug resistance by preventing the binding of the cardiac glycosides (Treschow 90 et al. 2007). In 2017, Agudelo et al. used "co-CRISPR" methods to target exon 4 of ATP1A1 91 concurrently with a second locus of interest in established human cell lines (Agudelo et al. 2017). 92 By selecting ATP1A1 mutations using ouabain, the percentage of cells harboring an INDEL at the 93 second locus increased (Agudelo et al. 2017). However, the co-CRISPR strategy was not applied to human iPSCs and in the cell lines that were tested the HDR rate was not significantly improved.

Here, we report the use of a CRISPR-Cas9 editing system that targets intron 4 of ATP1A1 in human iPSCs. By introducing a repair template to substitute the two N-terminal amino acids Q118 and N129, we specifically created an HDR-directed mutation that confers resistance to cardiac glycosides. We then applied different types of repair templates to investigate the efficiency of HDR. Furthermore, by co-targeting with the second locus of interest, we examined the efficiency of HDR-directed mutation after selection with the cardiac glycoside digoxin.

\section{Materials \& Methods}

\section{Construction of CRISPR/Cas9 plasmids and donor templates}

105

106

107

CRISPR guide RNAs were designed obeying the NGG PAM sequence rule. The construction of CRISPR plasmids followed the protocol established by Ran at al. in 2013 (Ran et al. 2013). To generate CRISPR construct targeting ATP1Al, a guide sequence (5'GAGTTCTGTAATTCAGCATA-3') was cloned into PX459 pSpCas9(BB)-2A-Puro plasmid following the fast digest protocol (Ran et al. 2013). The gRNAs targeting the other locus were cloned in to PX459 via the same procedure and the sequences of gRNAs were listed as below: (DGUOK exon1: 5'-CGAAGGCTCTCCATCGAAGG-3'; DGUOK exon4: 5'CATCGAGTGGCATATCTATC-3'; POLG exon10: 5'-ATGCAGGGTCGTCTAGCCGG-3'; GATA6 exon4: 5'-TTATGGCGCAGAAACGCCG-3'; RYR2B exon50: 5'TGACAGGGTCTATGGGATTG-3'). Single-stranded oligodeoxynucleotides (ssODNs) were obtained as Ultramer ${ }^{\circledR}$ DNA oligonucleotides (IDT, IL, US). Designed double-stranded DNAs were generated through GeneArt ${ }^{\mathrm{TM}}$ synthesis with the addition of restriction enzyme cutting sequences at both ends (Thermo Fisher Scientific, MA, US). To construct donor plasmids, dsDNAs from GeneArt ${ }^{\mathrm{TM}}$ synthesis were cut with restriction enzymes and cloned into pBlueScript II $\mathrm{KS}(+)$ 
119 (Addgene, MA, US). The sequences from all constructs were sequenced and verified before 120 proceeding with the CRISPR experiment. Donor templates for each targeted locus are listed in 121 Supplementary Table1.

122

\section{Cell lines, cell culture and electroporation}

124 Two human induced pluripotent stem cell lines, K3 hiPSCs (Si-Tayeb et al. 2010) and SV20

125

126

127

128

129

130

131

132

133

134

135

136

137

138

139

140

141

142

143

144

145

146

147

148

149

150

151

152

153

154

155

156

157

158 hiPSCs (Yang et al. 2015), which were characterized in previous studies, were used for this study. Provenance of all cell lines and use in these experiments were approved by the MUSC Stem Cell Research Oversight Committee protocol \#8. Cells were cultured in mTeSR feeder-free defined medium (Ludwig et al. 2006) supplemented with $4 \mathrm{ng} / \mathrm{mL}$ zebrafish basic fibroblast growth factor. All iPSCs were maintained as colonies on E-cadherin-IgG fusion protein matrix at $37{ }^{\circ} \mathrm{C}$ with $4 \%$ $\mathrm{O}_{2} / 5 \% \mathrm{CO}_{2}$ (Nagaoka \& Duncan 2010). Electroporation of plasmids and ssODNs used electroporator ECM630 (BTX, MA, US) following the manufacturer's instruction. Briefly, iPSCs were expanded until $80 \%$ confluence on one $100 \mathrm{~mm}$ dish to give approximately $4 \times 10^{7}$ cells and were harvested in small clumps using $0.05 \%$ EDTA. Cells were mixed with purified DNAs in 4 $\mathrm{mm}$ electroporation cuvette and immediately electroporated at 250 volts $/ 200 \Omega / 700 \mu \mathrm{F}$. Transfected cells were cultured onto $100 \mathrm{~mm}$ dish coated with Matrigel ( $2 \mathrm{mg} / \mathrm{ml}$ ) (Invitrogen, MA, US) in mTeSR medium supplemented with $4 \mathrm{ng} / \mathrm{mL}$ zebrafish basic fibroblast growth factor and $10 \mu \mathrm{M}$ of Y27632 (STEMCELL Technologies, VBC, CA) for 24 hours. For ATP1A1 co-targeting, cells were transfected with PX459 plasmids containing guide sequence of ATP1A1 and second gene of interest, along with linearized donor plasmids following the electroporation protocol above. After 24 hours of Y27632 treatment, transfected cells were selected by culturing with $1 \mu \mathrm{g} / \mathrm{mL}$ of puromycin for 48 hours. The survived cells were then recovered for an extra 48 hours with the normal mTeSR medium before digoxin selection. To obtain digoxin-resistant cells, $1 \mu \mathrm{M}$ of digoxin (Sigma, MO, US) was added on to the medium for 72 hours with medium changes performed on a daily basis. The surviving cells were harvested for genomic DNA extraction.

\section{RFLP and TIDE(R) analysis for genome modification}

The genomic DNA of iPSCs was harvested using QuickExtract ${ }^{\mathrm{TM}}$ DNA extraction solution following the manufacturer's instruction (Epicentre, Illumina, WI, US). Each single cell colony or the whole population of cells in a $100 \mathrm{~mm}$ dish were harvested in $50 \mu \mathrm{L}$ or $1 \mathrm{~mL}$ of QuickExtract reagent, respectively. Out-out PCR amplification was performed using Herculase II fusion DNA polymerase (Agilent, CA, US) with the following conditions: $\left(95^{\circ} \mathrm{C}\right.$ for $5 \mathrm{mins} ; 40 \times$ : $95^{\circ} \mathrm{C}$ for 30 $\mathrm{s}, 60^{\circ} \mathrm{C}$ for $30 \mathrm{~s}, 72^{\circ} \mathrm{C}$ for $30 \mathrm{~s} ; 72^{\circ} \mathrm{C}$ for 5 mins). PCR primers were located at least $100 \mathrm{bp}$ outside of donor templates region to prevent amplifying donor template. Primers for each targeted locus are listed in Supplementary Table 2. For RFLP analysis, PCR amplicons were purified via QIAquick PCR Purification Kit (Qiagen, MD, US), and $400 \mathrm{ng}$ of purified amplicons were digested EcoRI or NruI, respectively. Digested DNA was then separated on a 2\% agarose gel stained with ethidium bromide. For TIDE(R) analysis, purified PCR products were sequenced by Retrogen (CA, US), and the sequence signal of the experimental group was compared with wild- 
159

160

161

162

163

164

165

166

167

168

169

170

171

172

173

174

175

176

177

178

179

180

181

182

183

184

185

186

187

188

189

190

191

192

193

194

195

196

197

198

type or donor template as negative or reference control, respectively. TIDE(R) data analysis is described by Brinkman at al. in 2018 (Brinkman et al. 2018), and a free web tool is available at http://tide.nki.nl.

\section{Immunostaining and cell viability assay}

Wild-type and $A T P 1 A 1^{Q 118 R / N 129 D}$ iPSCs were treated with 15.625, 31.25, 62.5, 125, 250, 500, and $1000 \mathrm{nM}$ of digoxin and ouabain for 72 hours in a 96-well plate coated with Matrigel $(2 \mathrm{mg} / \mathrm{ml})$ (Invitrogen, MA, US). A cell viability assay was performed using CellTiter-Glo® luminescent cell viability assay kit following the manufacturer's instructions (Promega, WI, US). For nucleus immunostaining, cultured cells were fixed with $4 \%$ paraformaldehyde for 20 mins following $0.5 \%$ Triton X-100 treatment for 15 mins. Cells were blocked with 3\% bovine serum albumin in PBS for $30 \mathrm{mins}$ and incubated with DAPI $(1 \mu \mathrm{g} / \mathrm{ml})$ at room temperature for $30 \mathrm{mins}$. Fluorescence intensity was assessed with ZOE ${ }^{\text {TM }}$ Fluorescent Cell Imager (Bio-rad, CA, US). Experimental and control groups were processed identically.

\section{Statistical analysis}

Results generated by cell viability assay, survival colonies counting, and TIDE(R) analysis were expressed as mean SD. Data were analyzed by ANOVA followed by Tukey's or by Student's ttest, as appropriate. Statistical significance was achieved at $\mathrm{P}<.05$.

\section{Results}

\section{Designing CRISPR-Cas9 system to edit ATP1a1 locus}

Cardiac glycosides inhibit $\mathrm{Na}+/ \mathrm{K}+-\mathrm{ATPase}$ pump activity by binding to the ATP1A1 subunit. The blockage leads to the accumulation of $\mathrm{Ca}^{2+}$ and causes cell apoptosis (Riganti et al. 2011). Treschow et al. identified two amino acids in exon 4, Q118 and N129, that are responsible for the binding of cardiac glycosides to the $\mathrm{Na}+\mathrm{K}+$-ATPase (Treschow et al. 2007). With Q118R and N129D substitutions, cells became resistant to high doses of ouabain without affecting the function of the $\mathrm{Na}+/ \mathrm{K}+$-ATPase (Treschow et al. 2007). We, therefore, proposed to facilitate endogenous selection of targeted events in iPSCs using the CRISPR-Cas9 system to introduce the Q118 and N129 mutations into ATP1A1. We designed a guide RNA targeting intron 4 of ATP1A1, with the PAM site twenty base pairs downstream of exon 4 (Figure 1A). In order to generate the aminoacid substitution allele, we designed a 153 single-stranded oligodeoxynucleotides (ssODN) with the sequences that replace glutamine with arginine at position 118 and asparagine with aspartic acid at position 129 (Figure 1A). After transfecting both sp-Cas9-gRNA (px459-ATP1A1-intron4) and donor template into the cells and treating these cells with a high dose of digoxin, we collected the surviving clones for genotyping (Figure 1B). Using primers outside the targeted homology arms to perform PCR (out-out PCR) and restriction fragment length polymorphism (RFLP) analysis, we observed that all the cells that were resistant to digoxin or ouabain were successfully repaired through HDR, and both heterozygous and homozygous mutations were identified (Figure 
199

200

201

202

203

204

205

206

207

208

209

210

211

212

213

214

215

216

217

218

219

220

221

222

223

224

225

226

227

228

229

230

231

232

233

234

235

236

237

238

2A). The genotype was confirmed by genomic sequencing. In order to determine the optimum dosage of digoxin and ouabain, we performed a dose-response assay of both drugs on wild-type and ATP1A1 homozygous mutant iPSCs. We effectively eliminated all wild-type iPSCs at $1 \mu \mathrm{M}$ after 72 hours of exposure. In contrast to the parental iPSCs, homozygous ATP1A1 mutant iPSCs survived in the presence of either drug and were indistinguishable from untreated cells (Figure 2B to F). These results demonstrate that cardiac glycosides can be used to effectively identify HDRdriven mutations at the endogenous ATP1Al locus.

\section{Comparing the HDR efficiency while using different repair templates}

Although single-stranded oligodeoxynucleotides (ssODNs) have commonly been used as repair donor templates, the efficiency is generally low when using iPSCs (Radecke et al. 2010; Yoshimi et al. 2016). Double-strand DNAs with long homologous arms have been shown to improve the efficiency of introducing changes through genome editing in HEK293T cells (Song \& Stieger 2017). We therefore proposed to compare the efficiency of HDR-mediated mutagenesis between ssODNs and dsDNA at the ATP1A1 locus using endogenous selection. We co-transfected human iPSCs with px459-ATP1al-intron4 and donor template presented in the following formats: ssODNs, dsDNAs, or dsDNAs cloned into a plasmid. HDR-positive clones were then selected using a 72-hour exposure of $1 \mu \mathrm{M}$ digoxin (Figure 3A). As shown in Figure 3B, up to two positive clones were observed when co-transfected with 150 base-pair (bp) template either as ssODN, dsDNA, or linearized plasmid (Figure 3B). Strikingly, when cells were co-transfected with $400 \mathrm{bp}$ template either as dsDNA or linearized plasmid, the surviving colonies increased 11-fold and 35fold, respectively when compared to transfection using $150 \mathrm{bp}$ donors. These results indicate that double-stranded repair templates with $\geq 400$ bp length have a higher efficiency of generating HDRpositive cells. Moreover, assembling the dsDNA into a plasmid vector improved the effectiveness by a further $\sim 3$-fold (Figure 3B).

\section{Co-editing an independent locus by selecting for mutation of ATP1A1 in iPSCs}

To determine if selecting digoxin-resistant cells by modifying ATPlal locus increases the efficiency of HDR-driven events at a second locus of interest, we used the CRISPR-Cas9 system to co-target ATP1Al and deoxyguanosine kinase (DGUOK) gene in iPSCs. The DGUOK genetic alteration that we chose to introduce results in a loss of function mutation, DGUOK Q170X, which is observed in patients with Mitochondrial DNA Depletion Syndrome 3 (Hepatocerebral type) (MTDPS3); the patients with this syndrome commonly die from liver failure and suffer from progressive dysfunction of neural and muscular tissues (Mandel et al. 2001). Two different Human iPSC lines (SV20 and K3) (Jing et al. 2018; Si-Tayeb et al. 2010; Yang et al. 2015) were cotransfected with CRISPR plasmids targeting $A T P 1 A 1$ and DGUOK respectively, along with the linearized donor plasmids designed to introduce point mutations to each locus (Figure 4A). Transfected cells were then cultured until colonies were observed. At this point, cells were treated with or without $1 \mu \mathrm{M}$ of digoxin for 72 hours. In order to calculate the efficiency of HDR, genomic DNA was collected and PCR using primers outside the targeted homology arms of DGUOK exon4 
239 (out-out PCR) was performed to identify restriction fragment length polymorphisms (RFLP). 240 Amplicons were also sequenced and the frequency of indels and targeted mutations determined 241 using TIDE/TIDER analyses (Brinkman et al. 2018). Strikingly, we observed a significant increase

242 in HDR-mediated introduction of mutations after digoxin selection (Figure 4B). TIDER analysis 243 confirmed a two to three-fold increase in HDR-driven events from $4 \%$ to $13 \%$ in $\mathrm{K} 3$ and $8.3 \%$ to $24419 \%$ in SV20 iPSCs (Figure 4C). Moreover, the INDEL rate also dramatically increased from $24511.7 \%$ to $39 \%$ in K3 and $24.3 \%$ to $46.9 \%$ in SV20 iPSCs (Figure 4C). The results demonstrate that 246 after digoxin treatment to select cells with Q118 and N129 mutations in ATP1A1, both INDEL and HDR-driven targeting increased substantially at the second locus.

248

\section{HDR-driven event varies between genomic location}

To determine if the co-selection for the ATP1A1 $118 \mathrm{R} / \mathrm{N} 129 \mathrm{D}$ increases efficiency across different loci, we tested the same approach at other genes of interest, including RYR2, GATA6, and POLG, all of which are located on different chromosomes. We also targeted the DGUOK gene at a different independent locus. Each gene was targeted individually or along with ATPIA1. Transfected cells with or without digoxin selection were collected for out-out PCR, and INDELHDR rates were determined using TIDE/TIDER analysis. As shown in Table 1, the INDEL rates without digoxin selection ranged from $1.3 \%$ to $45.6 \%$, which is likely due to different inherent CRISPR guide targeting efficiencies. After digoxin selection, INDEL efficiency significantly increased among all targeted loci (Table 1). HDR rates remained low without digoxin selection, ranging from $0-4.18 \%$. After digoxin treatment, the cells showed an increase of HDR at both $D G U O K$ regions; however, this increase was not observed at the other targeted loci (Table 1) presumably due to the excessively low efficiency of HDR at these specific sites. In summary, the results indicate that the ATP1A1 co-targeting approach successfully selected targeted cells, and although HDR efficiency increases, the rate remains variable between loci.

\section{Discussion}

In the present study, we used CRISPR-Cas9 to edit the ATP1A1 locus in human iPSCs. The introduced mutations inhibited the binding of cardiac glycosides, thus conferring resistance to high dosage of this class of drug. More importantly, we were able to obtain genomic-modified clones that were specifically repaired by HDR after ouabain and digoxin selection. We showed that using $400 \mathrm{bp}$ dsDNA as the repair template increased HDR efficiency compared to 150bp ssODNs, while delivering the template in a linearized plasmid further improved HDR rates. Moreover, by cotargeting $A T P 1 A 1$ with a second locus of interest, we were able to increase both INDEL and HDR selection efficiency in two different iPSC lines. Although HDR-driven events varied between genomic loci, the overall efficiency of selecting genetically-modified clones increased. The optimization of the genome-editing strategy in human iPSCs provided by this study will pave the way for future disease modeling and gene therapy research. 
278 Optimizing repair templates to obtain increased HDR-driven mutations after double-strand breaks 279 has been well studied (Baker et al. 2017; Song \& Stieger 2017; Zhang et al. 2017). Due to 280 difficulties in generating ssODNs longer than $200 \mathrm{bp}$, researchers have used single strand DNA 281 with 30-70 base homology arms (HA) as donor template for small edits (Ran et al. 2013; 282 Richardson et al. 2016; Yang et al. 2013). The dsDNAs are generally used as the repair templates 283 for fragment insertions (Byrne et al. 2015; Urnov et al. 2005). In human iPSCs, the optimal HA 284 length is reported to be $\sim 2 \mathrm{~kb}$ (Byrne et al. 2015). Our study tested the efficiency of ssODN, 285 dsDNA and linearized plasmid for CRISPR-based genomic editing through HDR. We found that 286 homology arms that are less than $200 \mathrm{bp}$ showed no significant difference between all three forms 287 of donor DNA. We initially reasoned that this result might be due to low HDR efficiency in iPSCs, 288 as other studies showed that less than $2 \%$ of DSB repair was mediated through the HDR pathway 289 in stem cells (Yang et al. 2013). Strikingly, when we extended the length of dsDNA to $400 \mathrm{bp}$, 290 there was a dramatic increase in HDR efficiency. Our observations are similar to a previous report 291 showing that when the length of donor DNA was over 300 bp (150 bp HA), the efficiency of HDR292 driven events increased (Zhang et al. 2017). Moreover, when using linearized plasmids containing 293 the repair template in the center region, the number of positive clones was significantly higher than 294 non-plasmid dsDNA fragments. This observation may be due to DNA degradation after 295 transfecting into the cells, while linearized plasmids have higher stability because of the protection 296 inferred by the backbone sequence. The results are also consistent with another study showing that 297 using PCR products as repair templates are not as efficient as plasmid donors (Song \& Stieger 298 2017). Although applying long homology arm dsDNA as repair template is significantly improved 299 targeting, in this study it was only applied to ATP1A1. Whether this finding is suitable across 300 multiple loci remains to be rigorously determined; however, application of plasmids with large 301 homology domains at $D G U O K$ and $R Y R 2$ also enhanced our ability to retrieve mutations at these 302 sites.

303

The two major mechanisms to repair double-strand DNA breaks are NHEJ and HDR. Previous 305 studies have shown that HDR appears to be favored when cells are in the S-G2 phase of the cell cycle (Lieber 2010; Sonoda et al. 2006). Based on our observations, cells repaired by HDR on ATP1A1 after DSBs had both high activities of INDEL and HDR on the second locus, DGUOK, while other co-targeted genes didn't show significant improvement on HDR. Given that we did not attempt cell cycle synchronization, the observed differences in efficiencies are likely independent of the cell-cycle. Our data are also consistent with Agudelo's study that after ouabain selection, INDEL rate significantly increased on the second targeted locus (Agudelo et al. 2017). 312 Nevertheless, it is still unknown why selecting cells with HDR-driven mechanism on ATP1A1 313 enriched the overall mutagenesis population on the second locus. Recently, chromatin accessibility 314 has been found to be associated with CRISPR-Cas9 efficiency in zebrafish. The authors of that 315 study indicated that the open chromatin is more likely to be targeted by CRISPR-Cas9 (Uusi316 Makela et al. 2018). In the future, it would be interesting to investigate whether co-targeting with 
317 ATPIA1 and treating with digoxin preferentially selects cells that have greater chromatin

318

319

320

321

322

323

324

325

326

327

328

329

330

331

332

333

334

335

336

337

338

339

340

341

342

343

344

345

346

347

348

349

350

351

352

353

354

355

356

accessibility, and are therefore more susceptible to genome editing.

Variations within the $\mathrm{Na}^{+} / \mathrm{K}^{+}$-ATPase that confer resistance to cardiac glycoside have been found to occur naturally across species, including insects, metazoans, and rats (El-Mallakh et al. 2019; Perne et al. 2009; Ujvari et al. 2015). Q118R and N129D mutations in ATP1A1 have been rigorously examined and found to have no impact on $\mathrm{Na}^{+} / \mathrm{K}^{+}$-ATPase activity (Price et al. 1990). Instead the Q118R and N129D variations simply prevent the stable association of the glycoside with extracellular domain of the $\mathrm{Na}^{+} / \mathrm{K}^{+}$-ATPase. Therefore, ATP1A1 Q118R;N129D to confer resistance to glycoside toxicity should have no impact on cellular function. However, as is the case with all drug selection, there is a remote chance that conferring resistance to glycosides could have unintended consequences that should be considered (Askari 2019).

We have described one use for the endogenous selection approach which is to optimize conditions needed for efficient targeting. However, one could envision multiple uses for the same approach. strategy should be useful. For example, selection of glycoside resistance could be used to efficiently generate allelic variations in iPSCs that would facilitate disease modeling, ex vivo geneediting therapy, and drug discovery (Corbett \& Duncan 2019; Lee et al. 2020). Also, having an additional endogenous selectable allele will be helpful when there is a need to generate iPSC lines either with multiple variations within the same cells, revertant alleles, or rescue constructs.

Editing genomic DNA in iPSCs is challenging, not only due to relatively low HDR/INDEL rates, but also the colony-style culture methods which limit the capacity for single-cell sorting selection. Scientists have reported several strategies to address this challenge, including modifying medium, pre-treating with small molecules, and overexpressing anti-apoptotic genes such as BCL2 to increase cell viability during genome editing (Byrne \& Church 2015; Chen \& Pruett-Miller 2018; Gonzalez et al. 2014; Li et al. 2018). To improve the selection efficiency, researchers have also showed a significant increase on HDR/INDEL rate by introducing excisable markers such as PiggyBac or Cre/lox system; however, both these methods significantly increase the time required to generate the mutations of interest (Wang et al. 2017; Zhu et al. 2015).

\section{Conclusions}

We present a study using CRISPR-Cas9 to edit the ATP1A1 locus in human iPSCs and determining that introducing 400bp dsDNA repair template increased HDR efficiency compared to $150 \mathrm{bp}$ ssODNs. Moreover, we observed that by co-targeting ATP1A1 with a second locus of interest, the INDEL and HDR selection efficiency were improved in two different iPSC lines. Our described approach, using a one-step co-targeting strategy with a longer dsDNA repair template,

Peer) reviewing PDF | (2020:02:45862:1:1:NEW 2 Apr 2020) 
357

358

359

360

361

362

363

364

365

366

367

368

369

370

371

372

373

374

375

376

377

378

379

380

381

382

383

384

385

386

387

388

389

390

391

392

393

394

395

396

397

398

will shorten the period of selection process and increase both HDR/INDEL rate. $\mathrm{The}^{\mathrm{Na}} \mathrm{Na}^{+}$ channel is thought to be critical for maintaining cell osmolarity, thus the disruption of ionexchange ability results in cell death (Pierre \& Xie 2006). Since ATP1A1 mutation described here only affects the binding of glycosides and does not affect the function of $\mathrm{Na}^{+} / \mathrm{K}^{+}$pump, the approach has the advantage of being of marker-free selection that is suitable for clinical application.

\section{Acknowledgements}

We thank Dr. Morad and Dr. Yamaguchi for kindly providing CRISPR/Cas9 plasmid and donor templates targeting RYR2. We thank Drs. Pournasr, Jing, and Furio, for guidance and manuscript editing.

\section{References}

Agudelo D, Duringer A, Bozoyan L, Huard CC, Carter S, Loehr J, Synodinou D, Drouin M, Salsman J, Dellaire G, Laganiere J, and Doyon Y. 2017. Marker-free coselection for CRISPR-driven genome editing in human cells. Nat Methods 14:615-620. $10.1038 /$ nmeth. 4265

Askari A. 2019. The sodium pump and digitalis drugs: Dogmas and fallacies. Pharmacol Res Perspect 7:e00505. 10.1002/prp2.505

Baker O, Tsurkan S, Fu J, Klink B, Rump A, Obst M, Kranz A, Schrock E, Anastassiadis K, and Stewart AF. 2017. The contribution of homology arms to nuclease-assisted genome engineering. Nucleic Acids Res 45:8105-8115. 10.1093/nar/gkx497

Bapteste E, and Philippe H. 2002. The potential value of indels as phylogenetic markers: position of trichomonads as a case study. Mol Biol Evol 19:972-977. 10.1093/oxfordjournals.molbev.a004156

Belinky F, Cohen O, and Huchon D. 2010. Large-scale parsimony analysis of metazoan indels in protein-coding genes. Mol Biol Evol 27:441-451. 10.1093/molbev/msp263

Belusa R, Aizman O, Andersson RM, and Aperia A. 2002. Changes in $\mathrm{Na}(+)-\mathrm{K}(+)-A T P a s e$ activity influence cell attachment to fibronectin. Am J Physiol Cell Physiol 282:C302-309. 10.1152/ajpcell.00117.2001

Ben Jehuda R, Shemer Y, and Binah O. 2018. Genome Editing in Induced Pluripotent Stem Cells using CRISPR/Cas9. Stem Cell Rev 14:323-336. 10.1007/s12015-018-9811-3

Brinkman EK, Kousholt AN, Harmsen T, Leemans C, Chen T, Jonkers J, and van Steensel B. 2018. Easy quantification of template-directed CRISPR/Cas9 editing. Nucleic Acids Res 46:e58. 10.1093/nar/gky164 
399

400

401

402

403

404

405

406

407

408

409

410

411

412

413

414

415

416

417

418

419

420

421

422

423

424

425

426

427

428

429

430

431

432

433

434

435

436

437

438

439

440

441

442

443

444

Byrne SM, and Church GM. 2015. Crispr-mediated Gene Targeting of Human Induced Pluripotent Stem Cells. Curr Protoc Stem Cell Biol 35:5A 8 1-22. 10.1002/9780470151808.sc05a08s35

Byrne SM, Ortiz L, Mali P, Aach J, and Church GM. 2015. Multi-kilobase homozygous targeted gene replacement in human induced pluripotent stem cells. Nucleic Acids Res 43:e21. 10.1093/nar/gku1246

Chen F, Pruett-Miller SM, Huang Y, Gjoka M, Duda K, Taunton J, Collingwood TN, Frodin M, and Davis GD. 2011. High-frequency genome editing using ssDNA oligonucleotides with zinc-finger nucleases. Nat Methods 8:753-755. 10.1038/nmeth.1653

Chen YH, and Pruett-Miller SM. 2018. Improving single-cell cloning workflow for gene editing in human pluripotent stem cells. Stem Cell Res 31:186-192. 10.1016/j.scr.2018.08.003

Chu VT, Weber T, Wefers B, Wurst W, Sander S, Rajewsky K, and Kuhn R. 2015. Increasing the efficiency of homology-directed repair for CRISPR-Cas9-induced precise gene editing in mammalian cells. Nat Biotechnol 33:543-548. 10.1038/nbt.3198

Corbett JL, and Duncan SA. 2019. iPSC-Derived Hepatocytes as a Platform for Disease Modeling and Drug Discovery. Front Med (Lausanne) 6:265. 10.3389/fmed.2019.00265

Cornu TI, Mussolino C, and Cathomen T. 2017. Refining strategies to translate genome editing to the clinic. Nat Med 23:415-423. 10.1038/nm.4313

Deverman BE, Ravina BM, Bankiewicz KS, Paul SM, and Sah DWY. 2018. Gene therapy for neurological disorders: progress and prospects. Nat Rev Drug Discov. 10.1038/nrd.2018.158

El-Mallakh RS, Brar KS, and Yeruva RR. 2019. Cardiac Glycosides in Human Physiology and Disease: Update for Entomologists. Insects 10. 10.3390/insects 10040102

Fu Y, Foden JA, Khayter C, Maeder ML, Reyon D, Joung JK, and Sander JD. 2013. Highfrequency off-target mutagenesis induced by CRISPR-Cas nucleases in human cells. Nat Biotechnol 31:822-826. 10.1038/nbt.2623

Gaj T, Gersbach CA, and Barbas CF, 3rd. 2013. ZFN, TALEN, and CRISPR/Cas-based methods for genome engineering. Trends Biotechnol 31:397-405. 10.1016/j.tibtech.2013.04.004

Gonzalez F, Zhu Z, Shi ZD, Lelli K, Verma N, Li QV, and Huangfu D. 2014. An iCRISPR platform for rapid, multiplexable, and inducible genome editing in human pluripotent stem cells. Cell Stem Cell 15:215-226. 10.1016/j.stem.2014.05.018

Guo Q, Mintier G, Ma-Edmonds M, Storton D, Wang X, Xiao X, Kienzle B, Zhao D, and Feder JN. 2018. 'Cold shock' increases the frequency of homology directed repair gene editing in induced pluripotent stem cells. Sci Rep 8:2080. 10.1038/s41598-018-20358-5

Hsu PD, Scott DA, Weinstein JA, Ran FA, Konermann S, Agarwala V, Li Y, Fine EJ, Wu X, Shalem O, Cradick TJ, Marraffini LA, Bao G, and Zhang F. 2013. DNA targeting specificity of RNA-guided Cas9 nucleases. Nat Biotechnol 31:827-832. 10.1038/nbt.2647

Ihry RJ, Worringer KA, Salick MR, Frias E, Ho D, Theriault K, Kommineni S, Chen J, Sondey M, Ye C, Randhawa R, Kulkarni T, Yang Z, McAllister G, Russ C, Reece-Hoyes J, Forrester W, Hoffman GR, Dolmetsch R, and Kaykas A. 2018. p53 inhibits CRISPR-Cas9 engineering in human pluripotent stem cells. Nat Med 24:939-946. 10.1038/s41591-0180050-6

Jing R, Corbett JL, Cai J, Beeson GC, Beeson CC, Chan SS, Dimmock DP, Lazcares L, Geurts AM, Lemasters JJ, and Duncan SA. 2018. A Screen Using iPSC-Derived Hepatocytes Reveals $\mathrm{NAD}(+)$ as a Potential Treatment for mtDNA Depletion Syndrome. Cell Rep 25:1469-1484 e1465. 10.1016/j.celrep.2018.10.036

Peer) reviewing PDF | (2020:02:45862:1:1:NEW 2 Apr 2020) 
445 Johnson RD, and Jasin M. 2001. Double-strand-break-induced homologous recombination in

446

447

448

449

450

451

452

453

454

455

456

457

458

459

460

461

462

463

464

465

466

467

468

469

470

471

472

473

474

475

476

477

478

479

480

481

482

483

484

485

486

487

488

489

490 mammalian cells. Biochem Soc Trans 29:196-201.

Joung JK, and Sander JD. 2013. TALENs: a widely applicable technology for targeted genome editing. Nat Rev Mol Cell Biol 14:49-55. 10.1038/nrm3486

Kanaar R, Hoeijmakers JH, and van Gent DC. 1998. Molecular mechanisms of DNA double strand break repair. Trends Cell Biol 8:483-489.

Lee J, Bayarsaikhan D, Bayarsaikhan G, Kim JS, Schwarzbach E, and Lee B. 2020. Recent advances in genome editing of stem cells for drug discovery and therapeutic application. Pharmacol Ther:107501. 10.1016/j.pharmthera.2020.107501

Li XL, Li GH, Fu J, Fu YW, Zhang L, Chen W, Arakaki C, Zhang JP, Wen W, Zhao M, Chen WV, Botimer GD, Baylink D, Aranda L, Choi H, Bechar R, Talbot P, Sun CK, Cheng T, and Zhang XB. 2018. Highly efficient genome editing via CRISPR-Cas9 in human pluripotent stem cells is achieved by transient BCL-XL overexpression. Nucleic Acids Res. 10.1093/nar/gky804

Lieber MR. 2010. The mechanism of double-strand DNA break repair by the nonhomologous DNA end-joining pathway. Annu Rev Biochem 79:181-211. 10.1146/annurev.biochem.052308.093131

Lin YT, Wang CK, Yang SC, Hsu SC, Lin H, Chang FP, Kuo TC, Shen CN, Chiang PM, Hsiao M, Lu FL, and Lu J. 2017. Elimination of undifferentiated human embryonic stem cells by cardiac glycosides. Sci Rep 7:5289. 10.1038/s41598-017-05616-2

Luan PT, Ryder OA, Davis H, Zhang YP, and Yu L. 2013. Incorporating indels as phylogenetic characters: impact for interfamilial relationships within Arctoidea (Mammalia: Carnivora). Mol Phylogenet Evol 66:748-756. 10.1016/j.ympev.2012.10.023

Ludwig TE, Bergendahl V, Levenstein ME, Yu J, Probasco MD, and Thomson JA. 2006. Feederindependent culture of human embryonic stem cells. Nat Methods 3:637-646. $10.1038 /$ nmeth902

Mali P, Yang L, Esvelt KM, Aach J, Guell M, DiCarlo JE, Norville JE, and Church GM. 2013. RNA-guided human genome engineering via Cas9. Science 339:823-826. $10.1126 /$ science. 1232033

Mandel H, Szargel R, Labay V, Elpeleg O, Saada A, Shalata A, Anbinder Y, Berkowitz D, Hartman C, Barak M, Eriksson S, and Cohen N. 2001. The deoxyguanosine kinase gene is mutated in individuals with depleted hepatocerebral mitochondrial DNA. Nat Genet 29:337-341. 10.1038/ng746

Martin RM, Ikeda K, Cromer MK, Uchida N, Nishimura T, Romano R, Tong AJ, Lemgart VT, Camarena J, Pavel-Dinu M, Sindhu C, Wiebking V, Vaidyanathan S, Dever DP, Bak RO, Laustsen A, Lesch BJ, Jakobsen MR, Sebastiano V, Nakauchi H, and Porteus MH. 2019. Highly Efficient and Marker-free Genome Editing of Human Pluripotent Stem Cells by CRISPR-Cas9 RNP and AAV6 Donor-Mediated Homologous Recombination. Cell Stem Cell 24:821-828 e825. 10.1016/j.stem.2019.04.001

McDonough AA, Velotta JB, Schwinger RH, Philipson KD, and Farley RA. 2002. The cardiac sodium pump: structure and function. Basic Res Cardiol 97 Suppl 1:I19-24.

Nagaoka M, and Duncan SA. 2010. Transcriptional control of hepatocyte differentiation. Prog Mol Biol Transl Sci 97:79-101. 10.1016/B978-0-12-385233-5.00003-9

Pattanayak V, Lin S, Guilinger JP, Ma E, Doudna JA, and Liu DR. 2013. High-throughput profiling of off-target DNA cleavage reveals RNA-programmed Cas9 nuclease specificity. Nat Biotechnol 31:839-843. 10.1038/nbt.2673 
491

492

493

494

495

496

497

498

499

500

501

502

503

504

505

506

507

508

509

510

511

512

513

514

515

516

517

518

519

520

521

522

523

524

525

526

527

528

529

530

531

532

533

534

535

536

Perne A, Muellner MK, Steinrueck M, Craig-Mueller N, Mayerhofer J, Schwarzinger I, Sloane M, Uras IZ, Hoermann G, Nijman SM, and Mayerhofer M. 2009. Cardiac glycosides induce cell death in human cells by inhibiting general protein synthesis. PLoS One 4:e8292. 10.1371/journal.pone.0008292

Pierre SV, and Xie Z. 2006. The Na,K-ATPase receptor complex: its organization and membership. Cell Biochem Biophys 46:303-316.

Price EM, Rice DA, and Lingrel JB. 1990. Structure-function studies of Na,K-ATPase. Sitedirected mutagenesis of the border residues from the $\mathrm{H} 1-\mathrm{H} 2$ extracellular domain of the alpha subunit. J Biol Chem 265:6638-6641.

Radecke S, Radecke F, Cathomen T, and Schwarz K. 2010. Zinc-finger nuclease-induced gene repair with oligodeoxynucleotides: wanted and unwanted target locus modifications. $\mathrm{Mol}$ Ther 18:743-753. 10.1038/mt.2009.304

Ran FA, Hsu PD, Wright J, Agarwala V, Scott DA, and Zhang F. 2013. Genome engineering using the CRISPR-Cas9 system. Nat Protoc 8:2281-2308. 10.1038/nprot.2013.143

Richardson CD, Ray GJ, DeWitt MA, Curie GL, and Corn JE. 2016. Enhancing homologydirected genome editing by catalytically active and inactive CRISPR-Cas9 using asymmetric donor DNA. Nat Biotechnol 34:339-344. 10.1038/nbt.3481

Riganti C, Campia I, Kopecka J, Gazzano E, Doublier S, Aldieri E, Bosia A, and Ghigo D. 2011. Pleiotropic effects of cardioactive glycosides. Curr Med Chem 18:872-885.

Robinton DA, and Daley GQ. 2012. The promise of induced pluripotent stem cells in research and therapy. Nature 481:295-305. 10.1038/nature10761

Saleh-Gohari N, and Helleday T. 2004. Conservative homologous recombination preferentially repairs DNA double-strand breaks in the S phase of the cell cycle in human cells. Nucleic Acids Res 32:3683-3688. 10.1093/nar/gkh703

Sander JD, and Joung JK. 2014. CRISPR-Cas systems for editing, regulating and targeting genomes. Nat Biotechnol 32:347-355. 10.1038/nbt.2842

Shim G, Kim D, Park GT, Jin H, Suh SK, and Oh YK. 2017. Therapeutic gene editing: delivery and regulatory perspectives. Acta Pharmacol Sin 38:738-753. 10.1038/aps.2017.2

Si-Tayeb K, Noto FK, Sepac A, Sedlic F, Bosnjak ZJ, Lough JW, and Duncan SA. 2010. Generation of human induced pluripotent stem cells by simple transient transfection of plasmid DNA encoding reprogramming factors. BMC Dev Biol 10:81. 10.1186/1471213X-10-81

Smith TW. 1984. The basic mechanism of inotropic action of digitalis glycosides. J Pharmacol 15 Suppl 1:35-51.

Song F, and Stieger K. 2017. Optimizing the DNA Donor Template for Homology-Directed Repair of Double-Strand Breaks. Mol Ther Nucleic Acids 7:53-60. 10.1016/j.omtn.2017.02.006

Sonoda E, Hochegger H, Saberi A, Taniguchi Y, and Takeda S. 2006. Differential usage of nonhomologous end-joining and homologous recombination in double strand break repair. DNA Repair (Amst) 5:1021-1029. 10.1016/j.dnarep.2006.05.022

Treschow A, Unger C, Aints A, Felldin U, Aschan J, and Dilber MS. 2007. OuaSelect, a novel ouabain-resistant human marker gene that allows efficient cell selection within $48 \mathrm{~h}$. Gene Ther 14:1564-1572. 10.1038/sj.gt.3303015

Ujvari B, Casewell NR, Sunagar K, Arbuckle K, Wuster W, Lo N, O'Meally D, Beckmann C, King GF, Deplazes E, and Madsen T. 2015. Widespread convergence in toxin resistance by predictable molecular evolution. Proc Natl Acad Sci U S A 112:11911-11916. 10.1073/pnas. 1511706112

Peer) reviewing PDF | (2020:02:45862:1:1:NEW 2 Apr 2020) 
537

538

539

540

541

542

543

544

545

546

547

548

549

550

551

552

553

554

555

556

557

558

559

560

561

562

563

564

565

566

567

568

569

570

571

572

573

574

575

576

Urnov FD, Miller JC, Lee YL, Beausejour CM, Rock JM, Augustus S, Jamieson AC, Porteus MH, Gregory PD, and Holmes MC. 2005. Highly efficient endogenous human gene correction using designed zinc-finger nucleases. Nature 435:646-651. 10.1038/nature03556

Urnov FD, Rebar EJ, Holmes MC, Zhang HS, and Gregory PD. 2010. Genome editing with engineered zinc finger nucleases. Nat Rev Genet 11:636-646. 10.1038/nrg2842

Uusi-Makela MIE, Barker HR, Bauerlein CA, Hakkinen T, Nykter M, and Ramet M. 2018. Chromatin accessibility is associated with CRISPR-Cas9 efficiency in the zebrafish (Danio rerio). PLoS One 13:e0196238. 10.1371/journal.pone.0196238

Valerie K, and Povirk LF. 2003. Regulation and mechanisms of mammalian double-strand break repair. Oncogene 22:5792-5812. 10.1038/sj.onc.1206679

Wang G, Yang L, Grishin D, Rios X, Ye LY, Hu Y, Li K, Zhang D, Church GM, and Pu WT. 2017. Efficient, footprint-free human iPSC genome editing by consolidation of Cas9/CRISPR and piggyBac technologies. Nat Protoc 12:88-103. 10.1038/nprot.2016.152

Wang H, Yang H, Shivalila CS, Dawlaty MM, Cheng AW, Zhang F, and Jaenisch R. 2013. Onestep generation of mice carrying mutations in multiple genes by CRISPR/Cas-mediated genome engineering. Cell 153:910-918. 10.1016/j.cell.2013.04.025

WareJoncas Z, Campbell JM, Martinez-Galvez G, Gendron WAC, Barry MA, Harris PC, Sussman CR, and Ekker SC. 2018. Precision gene editing technology and applications in nephrology. Nat Rev Nephrol. 10.1038/s41581-018-0047-x

Yang L, Guell M, Byrne S, Yang JL, De Los Angeles A, Mali P, Aach J, Kim-Kiselak C, Briggs AW, Rios X, Huang PY, Daley G, and Church G. 2013. Optimization of scarless human stem cell genome editing. Nucleic Acids Res 41:9049-9061. 10.1093/nar/gkt555

Yang W, Liu Y, Slovik KJ, Wu JC, Duncan SA, Rader DJ, and Morrisey EE. 2015. Generation of iPSCs as a Pooled Culture Using Magnetic Activated Cell Sorting of Newly Reprogrammed Cells. PLoS One 10:e0134995. 10.1371/journal.pone.0134995

Yoshimi K, Kunihiro Y, Kaneko T, Nagahora H, Voigt B, and Mashimo T. 2016. ssODN-mediated knock-in with CRISPR-Cas for large genomic regions in zygotes. Nat Commun 7:10431. 10.1038/ncomms 10431

Yu C, Liu Y, Ma T, Liu K, Xu S, Zhang Y, Liu H, La Russa M, Xie M, Ding S, and Qi LS. 2015. Small molecules enhance CRISPR genome editing in pluripotent stem cells. Cell Stem Cell 16:142-147. 10.1016/j.stem.2015.01.003

Zhang JP, Li XL, Li GH, Chen W, Arakaki C, Botimer GD, Baylink D, Zhang L, Wen W, Fu YW, Xu J, Chun N, Yuan W, Cheng T, and Zhang XB. 2017. Efficient precise knockin with a double cut HDR donor after CRISPR/Cas9-mediated double-stranded DNA cleavage. Genome Biol 18:35. 10.1186/s13059-017-1164-8

Zhu Z, Verma N, Gonzalez F, Shi ZD, and Huangfu D. 2015. A CRISPR/Cas-Mediated Selectionfree Knockin Strategy in Human Embryonic Stem Cells. Stem Cell Reports 4:1103-1111. 10.1016/j.stemcr.2015.04.016

Peer] reviewing PDF | (2020:02:45862:1:1:NEW 2 Apr 2020) 


\section{Figure 1}

\section{Design of CRISPR/Cas9 targeting ATP1A1}

(A) The design of ATP1A1 guide RNA and single-stranded oligonucleotide (SSODN) donor.

Guide RNA was designed to target intron 4 of the ATP1Al gene. The expected cut site is 3-4' nucleotides upstream of the Protospacer Adjacent Motif (PAM) sequence and 20 nucleotides downstream of the exon 4 region. The donor template was designed to introduce nucleotide substitutions that result in the replacement of Glutamine with Arginine (Q118R) and Asparagine with Aspartic acid (N129D). The EcoRI cut site was introduced to allow identification of positive clones. (B) The timeline of performing gene-editing on human iPSCs. Human iPSCs were transfected with a CRISPR/Cas9 plasmid targeting ATPIA1 intron 4 and a repair template through electroporation. Cells were treated with $10 \mu \mathrm{M}$ Y27632 for 24 hours following 48 hours of puromycin exposure to select transfected iPSCs. Cells were maintained for an extra 48 hours until colonies were observed and before administering digoxin for 72 hrs. Surviving cells were isolated and genotype determined by sequence analyses and PCR. 
A.

ATP1A1 genome

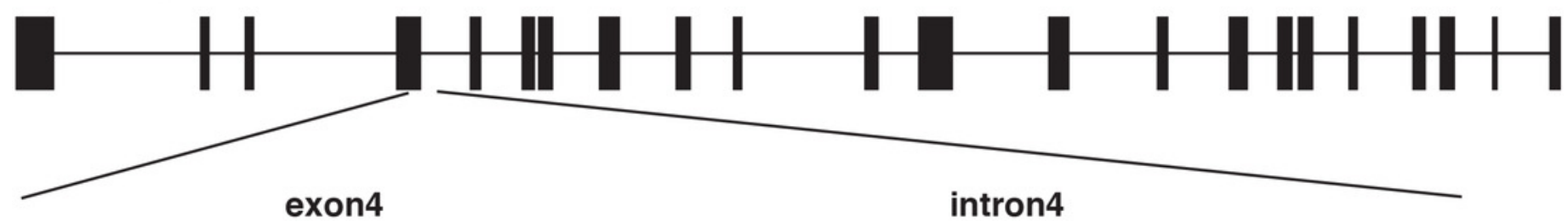

GAG GAA CCT CAA AAC GAT AAT ... gtgagttctgtaattcagcatatggatttgtagtacacatcaga

E $\quad$ E $\quad P \quad \underline{Q} \quad \mathrm{~N} \quad \mathrm{D} \quad \underline{\mathrm{N}}$

Q118 $\quad$ guide $\quad$ PAM

Q118R N129D ssODN

GAG GAA CCT CGG AAC GAT GAT ... gtgagttctggaattcagcatatggatttgtagtacacatcaga

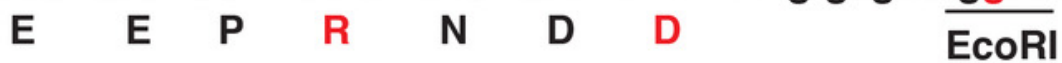

B.

Electroporation

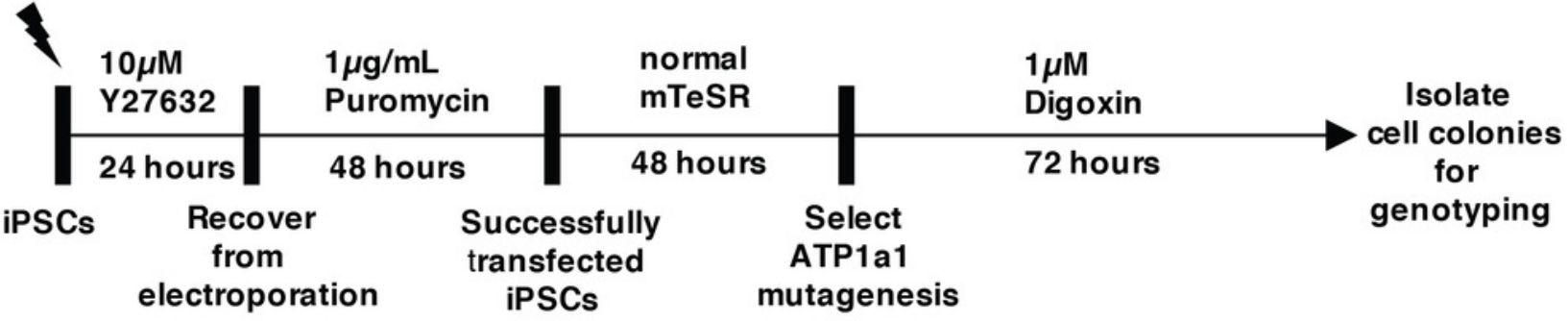




\section{Figure 2}

Introduction of ATP1A1 ${ }^{\text {Q118R/N129D }}$ in human iPSCs confers resistance to cardiac glycosides

(A) RFLP analysis of ATP1A1 mutation. Transfected iPSCs were selected through $1 \mu \mathrm{M}$ of digoxin exposure and genomic DNA from each individual colony was extracted for out-out PCR reaction. PCR amplicons were digested by EcoRI to identify homozygous and heterozygous mutations (for raw data see Figure S1). (B) Wild-type iPSCs and homozygous ATP1A1 mutants were exposed to 15.625, 31.25, 62.5, 125, 250, 500, and $1000 \mathrm{nM}$ of digoxin and ouabain for 72 hours. Cell viability was determined by luminescence assay ( $B$, for raw data see DataS1) and was confirmed by DAPI staining (C-F) (for uncropped images see figures S2-S5). The experiment was conducted in triplicate $(n=3)$ and data are shown as mean \pm SD. Statistical differences were determined by ANOVA followed by Tukey's test $(* p<$ .01). (Wild Type Oubain: $n=3, d f=8, F=5731.75, p=4.85 \times 10^{-29}$; Wild Type Digoxin: $n=3, d f=8$, $\left.F=37.33, p=1.17 \times 10^{-9}\right)$. 
A

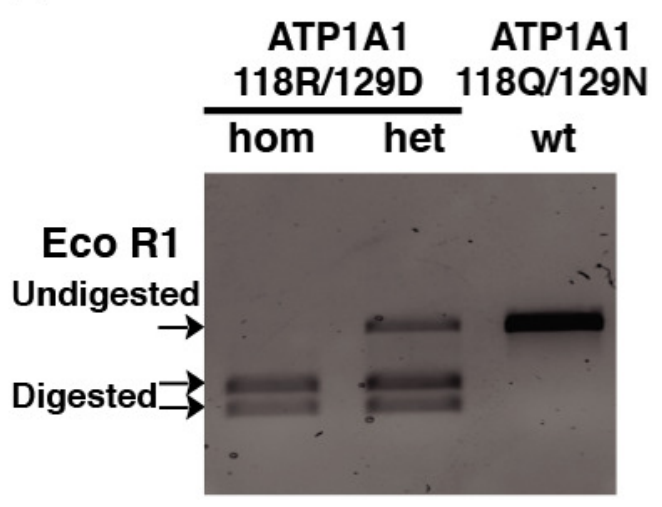

C ATP1A1(118Q/129N)

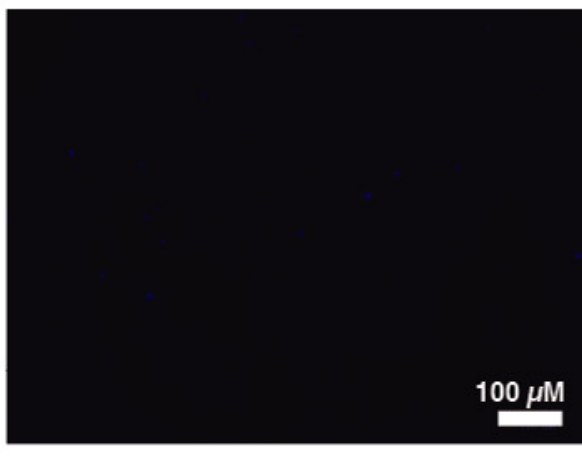

Digoxin

$(1 \mu \mathrm{M})$

B

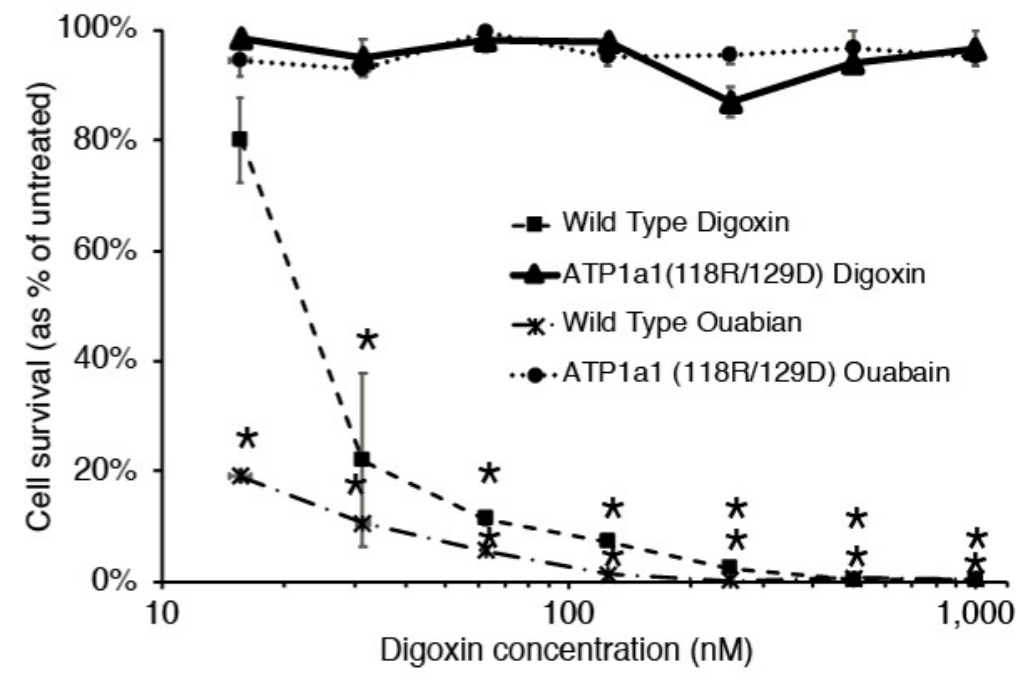

D ATP1A1(118R/129D)

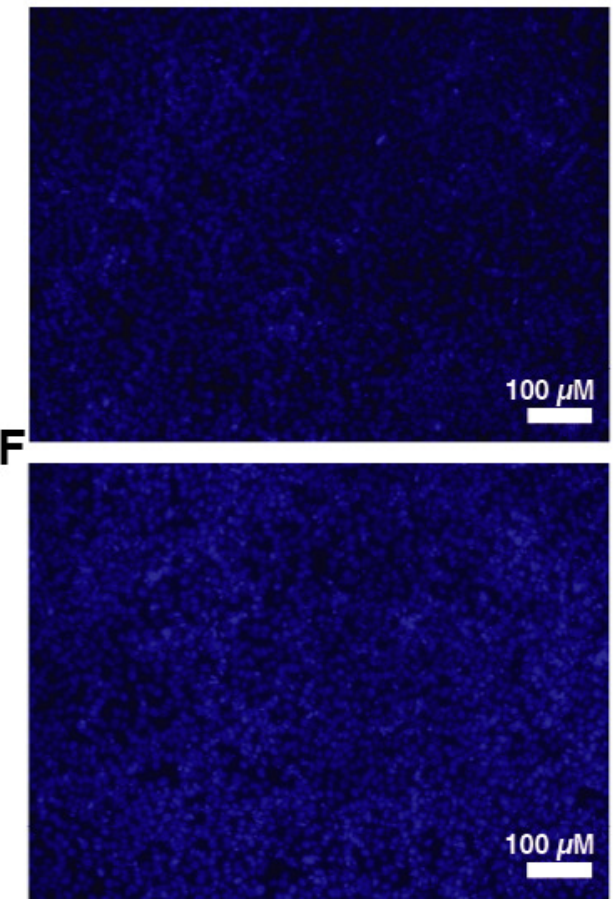




\section{Figure 3}

Provision of long HA dsDNA donor template during gene editing increases HDRmediated mutagenesis

(A) Diagram of approach used to edit ATP1A1 using different donor templates. $1 \times 10^{7}$ of human iPSCs were transfected with PX459 containing guide RNAs which target intron 4 via electroporation. Cells were also transfected with 0.01 nmole of ssODN, dsDNA, and linearized donor plasmid, respectively. Seven days after electroporation, cells were treated with $1 \mu \mathrm{M}$ of digoxin for 72 hours. (B) The number of surviving iPSC colonies for each group was counted. The experiment was conducted in triplicate $(n=3)$. Data are shown as mean \pm SD. Statistical differences were determined by ANOVA followed by Tukey's test $(* p<.05) .(n=3, d f=4$, $F=21.17, p=7.19 \times 10^{-5}$ )

A

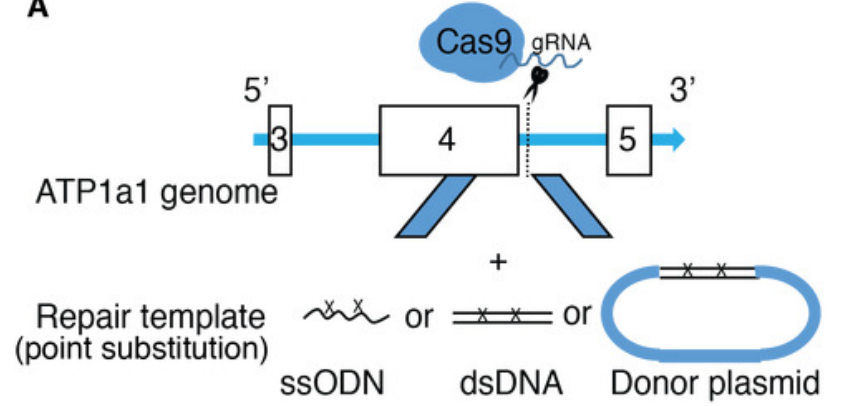

Survival cells

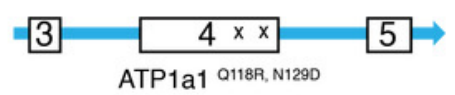

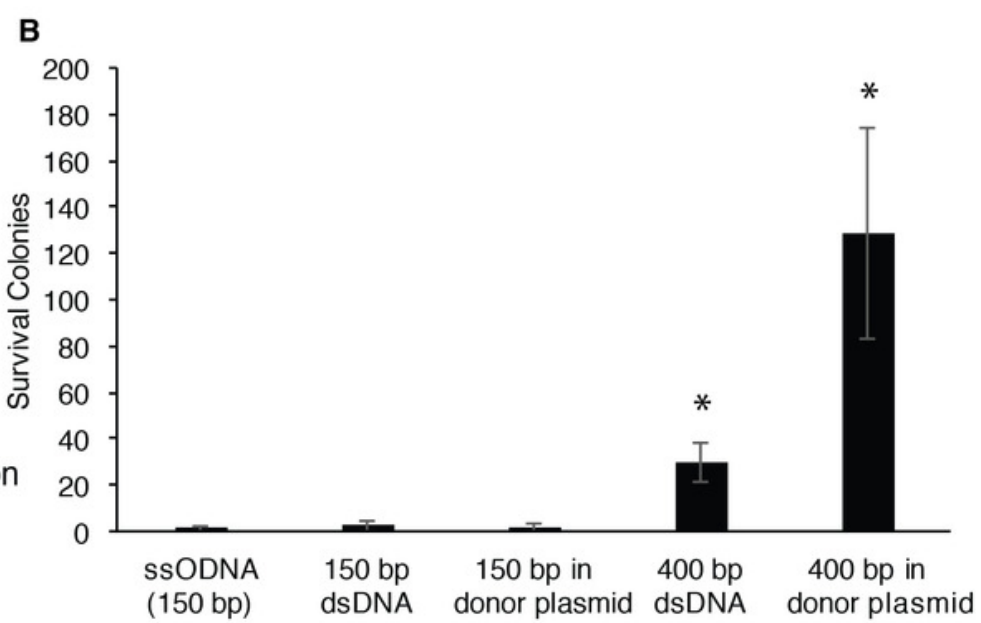




\section{Figure 4}

Selecting for mutation of ATPIAI by digoxin enriches HDR and INDEL-mediated mutagenesis at the DGUOK gene.

(A) Diagram of CRISPR co-targeting appraoch. Two CRISPR plasmids PX459 containing guide RNAs (ATPIA1 Intron 4 and DGUOK exon 4), and two linearized repair templates were transfected into two different iPSC lines (K3 and SV20). The DGUOK repair template introduced a Nrul restriction enzyme cutting sequence to facilitate RFLP analysis. Cell selection was demonstrated in figure 1B. (B) Cells with or without culture in $1 \mu \mathrm{M}$ digoxin were collected and genomic DNA was extracted from the whole population. RFLP analysis was performed to determine the level of enrichment of HDR-driven mutagenesis (for uncropped image see figure S6). (C) Out-out PCR amplicons from (B) were sequenced and subjected to TIDER analysis (for raw data see Data S2). The genome editing experiment was performed in five replicates $(n=5)$. Data are shown as mean $\pm S D$. Statistical differences between each two means (Digoxin-treated V.S. non-treated) were determined by student Ttest $(* p<.05, * * p<.01)$ (SV20 Indel: $p=5.1 \times 10^{-4}, K 3$ Indel: $p=3.34 \times 10^{-4}$, SV20 HDR: $p=1.33 \times 10^{-3}, K 3$ HDR: $\left.p=3.64 \times 10^{-4}\right)$. 
A ATP1A1 Intron4 gRNA

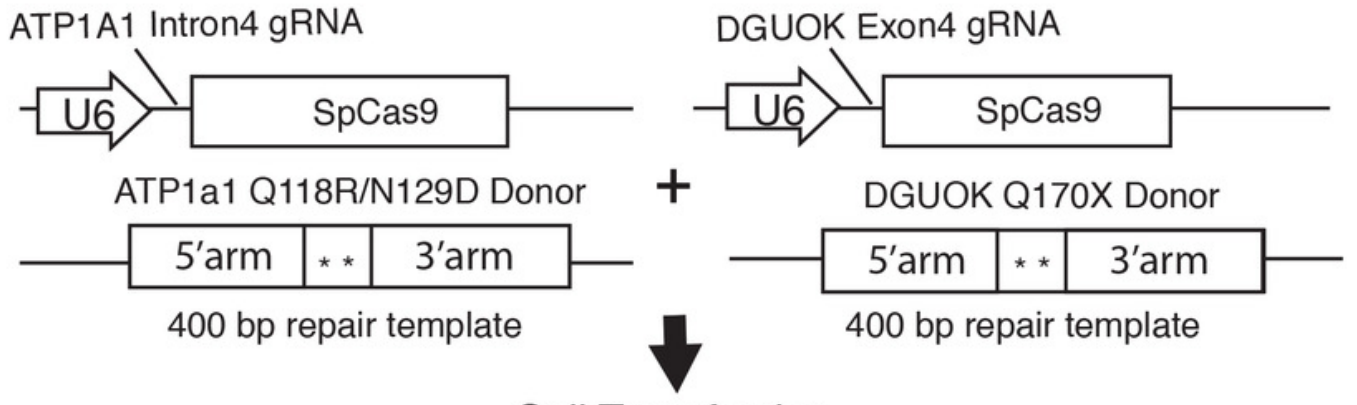

Cell Transfection

Digoxin selection

ATP1A1 Exon4 locus

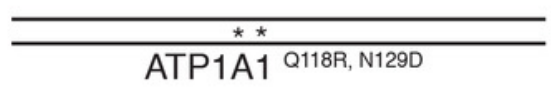

DGUOK Exon4 locus

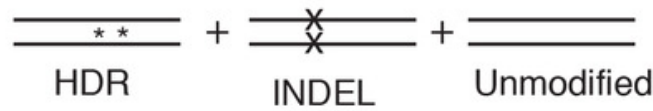

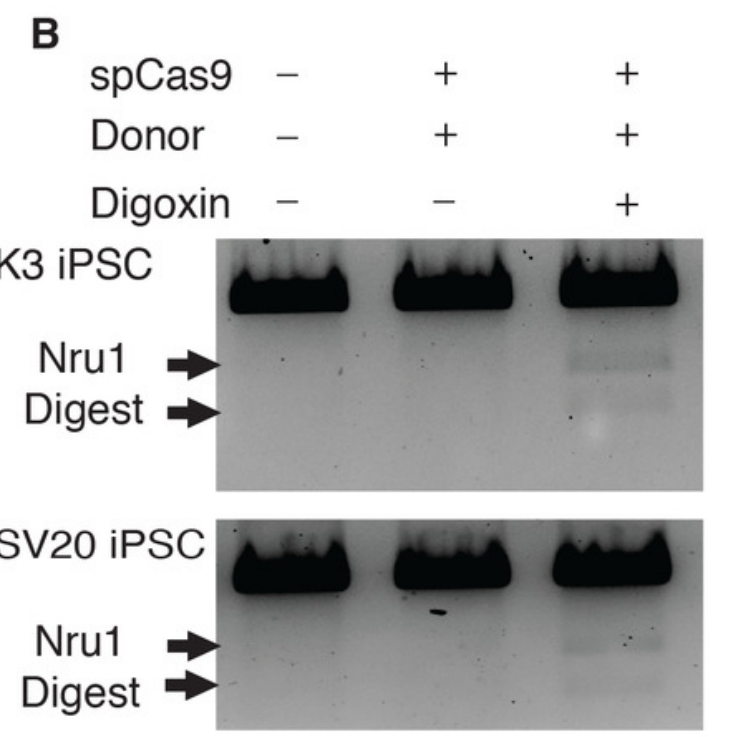

C $\star \star \star \quad$ TIDER analysis

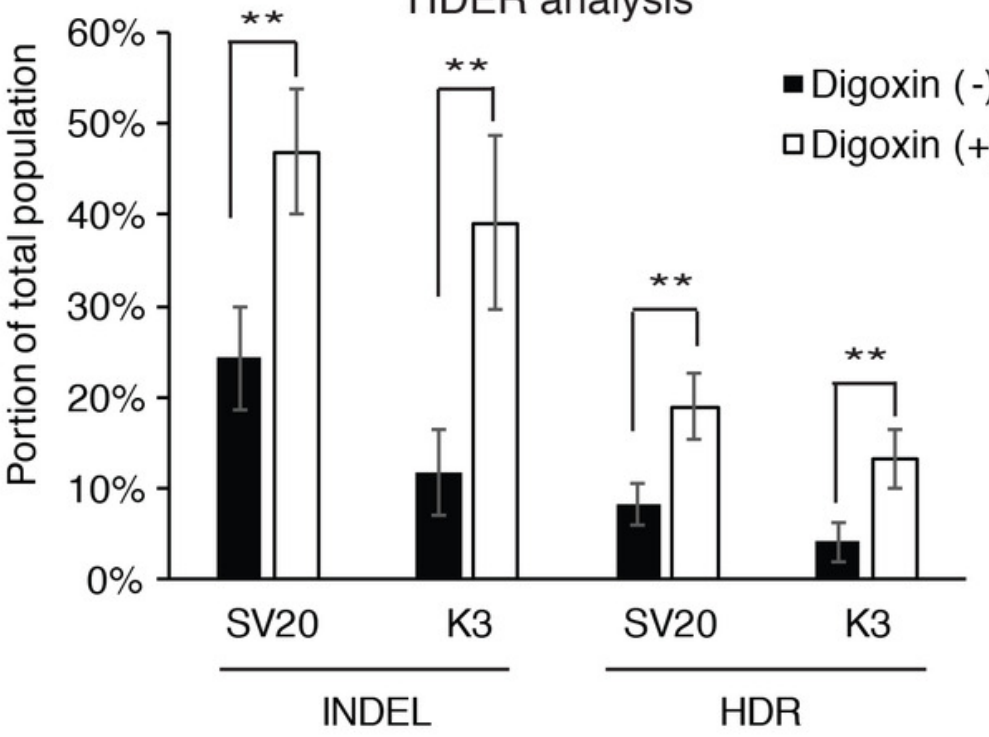




\section{Table $\mathbf{1}$ (on next page)}

The population of HDR and INDEL-mediated mutagenesis after co-targeting ATPIAI and a second locus of interest

Five different loci were targeted by CRISPR-Cas9 in human iPSCs along with two linear repair templates (ATPIA1 and a second locus of interest). After transfection cells were cultured in the presence or absence of digoxin selection, cells were collected and sequenced for TIDE(R) analysis. The percent population of INDELs and HDR events are shown. 
1

\begin{tabular}{l|l|ll|ll}
\multicolumn{5}{l}{ Indel } & HDR \\
\hline Gene & Chromosome & Digoxin (-) & Digoxin (+) & Digoxin (-) & Digoxin (+) \\
\hline DGUOK_EX1 & 2 & $16.53 \%$ & $43.10 \%$ & $0 \%$ & $10.85 \%$ \\
DGUOK_EX4 & 2 & $11.76 \%$ & $39.03 \%$ & $4.18 \%$ & $13.23 \%$ \\
RYR2_EX50 & 1 & $12.40 \%$ & $43.53 \%$ & $1.35 \%$ & $3.13 \%$ \\
GATA6_EX4 & 18 & $45.60 \%$ & $81.80 \%$ & $0.40 \%$ & $0 \%$ \\
PolG_EX10 & 15 & $1.38 \%$ & $25.33 \%$ & $0 \%$ & $0 \%$
\end{tabular}

